\title{
Cambio Climático: estrategias de gestión con el tiempo en contra...
}

\section{Climate change: management strategies racing against time...}

\author{
Mudanças climáticas: estratégias de gestão \\ com o tempo em contra...
}

Adriana Yepes - Mayorga ${ }^{1 *}$ Dra en Ciencias Biológicas de la Universidad de San Paulo, Instituto de Biociencias, Departamento de Botánica,
Apartado aéreo 11461, San Paulo, SP, Brasil.* Docente de la Facultad de Ciencias Básicas. Universidad de los Llanos.
Email: adyepes@yahoo.com

Recibido: octubre 10 de $2011 \quad$ Aprobado: marzo 21 de 2012

\section{Resumen}

El mundo debe enfrentarse a cambios drásticos del clima, en intensidad y magnitud. El cambio climático es favorecido por actividades humanas, lo que conlleva el desafío aún mayor de sustituir estereotipos estilo de vida y explotación de los recursos naturales en el futuro inmediato. Aquel modelo de desarrollo cortoplacista, basado en la extracción exhaustiva, al precio de la contaminación y degradación de los recursos naturales en función de la fabricación de bienes y servicios suntuarios, pone en riesgo condiciones básicas de bienestar a las generaciones actuales y futuras de otras regiones del planeta. Desde ya son percibidos nuevos regímenes de sequías y lluvias que golpean recursos públicos, así como a la base social y económica de los países, potencializando problemas económicos, ambientales y de salud pública en el caso de Latinoamérica.

En el siguiente documento se hará una revisión de lo que conocemos hasta ahora sobre el cambio climático y cómo el desarrollo de la sociedad ha favorecido este fenómeno. Se describirá la sinergia entre los procesos de adaptación y mitigación, las vulnerabilidades de nuestra región, así como las medidas de gestión propuestas por científicos, agencias y organismos internacionales para disminuir el impacto negativo de este fenómeno, junto con sugerencias de acciones que cada uno de nosotros tiene a su alcance.

Palabras Clave: vulnerabilidad; adaptación; mitigación; gestión ambiental; economía verde

\begin{abstract}
The world must face drastic climate change (i.e. re intensity and magnitude). Climate change is promoted by human activities leading to the even greater challenge of substituting stereotyped lifestyles and exploitation of natural resource in the immediate future. Such short-term development model based on exhaustive extraction, the price of contamination and degradation of natural resources regarding the fabrication of sumptuary goods and services puts basic conditions of wellbeing at risk for current and future generations from other regions of the planet. New patterns of drought and flooding dealing harsh blows to public resources are already being perceived, as well as countries' social and economic basis, promoting economic, environmental and public health problems in Latin-America.
\end{abstract}


The following document reviews what is known to date about climate change and how society's development has promoted such a phenomenon. The synergy between adaptation and mitigation and our region's vulnerability are described as well as the management measures proposed by scientists, agencies and international organisms for reducing such phenomenon's negative impact, together with suggestions for easily achievable action which can be taken by every one of us.

Key words: vulnerability, adaptation, mitigation, environmental management, green economy.

\begin{abstract}
Resumo
O mundo esta enfrentando mudanças radicais no clima, em intensidade e magnitude. As mudanças climáticas são fornecidas pelas atividades antropogênicas, o que leva a um desafio ainda maior: substituir estereótipos de vida e exploração de recursos naturais no futuro imediato. Aquele modelo de desenvolvimento a corto prazo, baseado na extração exaustiva, ao preço da contaminação e degradação dos recursos naturais em função da manufatura de bens e serviços de luxo, põe em risco as condições básicas de bem-estar das gerações presentes e futuras de outras regiões do planeta. Desde já são identificados novos padrões de chuvas e seca que atingem aos recursos públicos, assim como à base social e econômica da população, potencializando problemas econômicos, ambientais e de saúde pública em Latino América.

No presente artigo, será feita uma revisão do conhecimento até agora atingido sobre as Mudanças Climáticas, e de como o desenvolvimento econômico da sociedade favorece este fenômeno. A sinergia entre os processos de adaptação e mitigação será descrita, as vulnerabilidades da nossa região, assim como as medidas de gestão propostas pela comunidade cientista, agencias e organismos internacionais para diminuir o impacto negativo deste processo. Também serão propostas sugestões de ações que cada um de nós tem à mão pôr em prática.
\end{abstract}

Palavras chave: vulnerabilidade, adaptação, mitigação; gestão ambiental; economia verde

\begin{abstract}
Abreviaturas
$\mathrm{CO}_{2}$ : dióxido de carbono; CMNUCC: Conversión Marco de la Naciones Unidas sobre cambio climático; $\mathrm{CH}_{4}$ : $\mathrm{metano}$; CFC: clorofluorocarbonatos; GEI: gases de efecto invernadero; HCFC: hidro clorofluorocarbonatos; IPCC: Programa Intergubernamental para el cambio climático; Gton: gigatoneladas; $10^{15}$ gramos; MANUD: Marco de Asistencia al Desarrollo de las Naciones Unidas; $\mathrm{NH}_{4}$ : amonio; $\mathrm{NOx}$ : óxidos de nitrógeno; OMM: Organización Mundial de Meteorología; PK: Protocolo de Kioto; PNUMA: Programa de las Naciones Unidas para el medio ambiente (en inglés, UNEP, United Nations Environment Program); W: grupo de trabajo del IPCC; WI: grupo de trabajo I del IPCC, la ciencia del cambio climático los impactos; WII: grupo de trabajo II del IPCC, adaptaciones y vulnerabilidad; WIII: grupo de trabajo III del IPCC, mitigación
\end{abstract}

\section{Introducción}

Ante la ya inminente alteración en el clima a nivel mundial favorecida por las actividades humanas, no sólo la comunidad académica se muestra preocupada por entender cómo hemos conseguido modificar, si bien involuntariamente, los procesos naturales. En los países en vías de desarrollo, las comunidades menos favorecidas económicamente son especialmente las más vulnerables. Sin embargo, la intensidad de los eventos climáticos ha tocado todos niveles sociales a través de inundaciones, tormentas, ondas de calor, lo que demuestra que las previsiones hasta ahora tomadas no son suficientes.

La Conversión Marco de la Naciones Unidas sobre Cambio Climático (CMNUCC, 1992) definió Cambio Climático como los cambios en el clima originados en causas humanas. En los informes del IPCC ${ }^{1}$, cambio

IPCC Panel intergubernamental para el cambio climático climático se refiere a todo cambio en el clima, ya sea debido a la variabilidad natural o como resultado de la actividad humana (Parry et al., 2007). El presente artículo toma esta última acepción.

Los programas de gestión ante el cambio climático tienen el tiempo en su contra: mientras buscamos entender los mecanismos de los fenómenos naturales alterados, los científicos incorporan nuevos elementos a los complejos modelos, para así proponer medidas de mitigación y de adaptación que permitan mejorar la calidad de vida de las personas, lo que involucra acoplar las nuevas prácticas ambientales de forma armónica entre diferentes áreas de la administración pública (medio ambiente, hacienda, transporte) y privada. La sociedad conoce la vulnerabilidad de su medio ambiente, pero las autoridades que toman decisiones están preocupadas por su futuro inmediato, por lo que las acciones de largo aliento quedan aplazadas (Rodríguez-Becerra y Espinosa 2002). 
El cambio climático representa una oportunidad de revertir los déficits sociales acumulados en las diferentes regiones del planeta. Igualmente reitera la necesidad para que los países desarrollados avancen a un consenso, por cuanto ellos siguen detentando el control sobre las decisiones de carácter estratégico. Las propuestas de adaptación a este proceso implican participación de todos los grupos sociales, de las naciones y grupos económicos. Es necesario reivindicar el papel de la ciencia para conseguir entender la dinámica del clima en el planeta. Se requiere de la inclusión y apoyo financiero de los países emergentes para hacer más integral el desarrollo económico y evitar o corregir nacientes conflictos sociales. Aunque estamos aprendiendo sobre la marcha, ya hay experiencias exitosas viables, que requieren de apoyo político y económico, a las que bien podríamos darles una oportunidad.

\section{Ciencia del cambio climático. Contexto histórico.}

El clima en la Tierra ha experimentado diversas transformaciones a lo largo de la historia del planeta, que están relacionadas con aspectos externos como variaciones solares, orbitales, meteoritos (Crowley y North, 1988), y con aspectos internos como la composición de la atmósfera, las corrientes oceánicas, el campo magnético terrestre y las actividades humanas (Oreskes, 2004). El sistema climático de la Tierra es complejo e interactivo, ya que consta de elementos presentes en la atmósfera, la superficie terrestre y en los cuerpos de agua, además de los organismos que la habitamos (Yepes - Mayorga y Buckeridge, 2011).

Nuestro conocimiento sobre el clima se ha venido desarrollando a través de la propia historia de la ciencia. Como lo describe Le Treut et al. (2007), ya en el siglo XVII Edme Mariotte observó que la atmósfera se comporta como un invernadero, permitiendo el paso de radiación y reteniendo parte del calor. Esta observación fue complementada por Tyndall dos siglos después (1859), cuando evidenció que un aumento en la concentración de gases radioactivamente activos (como $\mathrm{CO}_{2}{ }^{2}, \mathrm{NH}_{4}{ }^{3}$ y agua) podrían producir cambios importantes en el clima. En este mismo sentido, Callendar (1938) usando cálculos matemáticos, estableció que al doblar la concentración de $\mathrm{CO}_{2}$ atmosférico, la temperatura media global aumentaría, asociando este fenómeno con la quema de combustibles fósiles y el efecto invernadero (Le Treut et al., 2007).

\footnotetext{
$\mathrm{CO}_{2}$ : dióxido de carbono

$\mathrm{NH}_{4}$ : amonio
}

\section{Dinámica Global del cambio climático}

La Revolución Industrial de la Europa del siglo XIX permitió cambios económicos de la sociedad al incorporar el uso de la máquina a vapor, haciendo más eficientes los sistemas industriales de producción. La locomotora y el barco a vapor complementaron el cuadro, facilitando los sistemas de transporte. Todos estos avances se consiguieron gracias al uso de combustibles fósiles, petróleo, y carbón mineral. También se aumentó la producción de carbón vegetal, (Martín, 1989), pero su producción ha significado un impacto directo sobre bosques porque requiere de grandes cantidades de madera. Así, rápida y erróneamente, la imagen de gigantescas chimeneas acompañadas de grandes emisiones de gases, fueron sinónimo de progreso. En la actualidad, la atmósfera recibe por año alrededor de 118 gigatoneladas (Gton) ${ }^{4}$ de $\mathrm{CO}_{2}$ procedentes de la quema de combustibles fósiles, del cambio de uso del suelo y quemas de bosques, de la respiración de las plantas y de la respiración del suelo. De este $\mathrm{CO}_{2}$ liberado a la atmósfera, los océanos y las plantas reabsorben alrededor de 113 Gton/año. De acuerdo a estos cálculos, existe un diferencial entre el $\mathrm{CO}_{2}$ calculado y el $\mathrm{CO}_{2}$ medido en la atmósfera, lo que pone de manifiesto nuestro vacío en el conocimiento del ciclo del carbono (Sampaio et al., 2008). Este diferencial anual entre el $\mathrm{CO}_{2}$ emitido y el absorbido, además del calentamiento global, ha conducido a cambios en los patrones de precipitación y del sistema atmosférico como un todo.

Junto a éstos, los problemas ambientales más serios han sido la deforestación, la pérdida de la diversidad, el empobrecimiento y degradación de los suelos, la contaminación y disminución de fuentes de agua dulce, así como la contaminación de los mares. El declive en la capa de ozono, los contaminantes orgánicos persistentes, así como organismos vivos modificados, también son consecuencia del desarrollo cortoplacista (Rodríguez- Becerra, 2005).

Desde la Conferencia de la Naciones Unidas sobre Recursos Naturales realizada en 1949, voces conservacionistas vienen haciendo un Ilamado a la sociedad para dar mayor atención al impacto que las prácticas de desarrollo tienen en el medio ambiente. En la Conferencia de Estocolmo de 1972 se acordaron convenciones globales periódicas y la incorporación de la gestión ambiental por cuenta del Estado. La primera conferencia mundial sobre el Clima fue realizada en 1979 en Ginebra, a la que sucedieron una serie de iniciativas a nivel mundial. En 1987, la agenda 
de la Conferencia de Estocolmo fue perfeccionada con el Reporte Brundtland, que definió el concepto de desarrollo sostenible en sus tres dimensiones: económico, social y ambiental. En el año 1988 el Programa de las Naciones Unidas para el Medio Ambiente (PNUMA) y la Organización Mundial de Meteorología (OMM) crearon el Programa Intergubernamental para el Cambio Climático (IPCC) para compilar la información científica, técnica y socioeconómica del cambio climático (Yepes-Mayorga y Buckeridge, 2011). En la Conferencia de las Naciones Unidas para el Medio Ambiente y Desarrollo de Río (1992) se hizo un Ilamado hacia el Desarrollo Sostenible con un plan de acción consignado en la Agenda 21. Con el Protocolo de Kyoto (ONU, 1997) ya se pretendía generar consenso para reducir las emisiones de gases de efecto invernadero (GEI) en los países responsables.

Luego de informes emitidos en 1990, 1996, 2001 y 2007, los tres grupos de trabajo (W) del IPCC compilaron investigaciones sobre la ciencia del cambio climático (WI), los impactos, adaptaciones y vulnerabilidad (WII), así como los aspectos de mitigación del mismo (WIII), estableciendo los lineamientos que gobiernos, instituciones privadas y públicas deberían considerar para establecer una política ambiental transversal a todas las esferas económicas, sociales y culturales (Solomon et al., 2007).

\section{Construcción de conceptos:}

Impactos, adaptación y vulnerabilidad

frente al cambio climático

Existen evidencias que relacionan las respuestas de los sistemas físicos y biológicos en la Tierra con el componente humano del proceso del calentamiento global. El aumento en las emisiones de gases con efecto invernadero (ver Tabla 1) y su concentración en la atmósfera ha llevado a un incremento en la temperatura. La temperatura media global de la superficie de la Tierra, que había sido relativamente estable en los últimos 1000 años, ha aumentado $0,75^{\circ} \mathrm{C}$ desde la era pre-industrial y un aumento de entre $0,5^{\circ} \mathrm{C}$ y $1.0^{\circ} \mathrm{C}$ es inevitable debido a las emisiones pasadas. Se espera un incremento adicional de $1.2^{\circ} \mathrm{C}$ a $6,4^{\circ} \mathrm{C}$ entre 2000 y 2100 , con un calentamiento mayor en áreas continentales que en los océanos y mayor en el Ártico que en el Trópico. Este evento ya ha generado fenómenos extremos como olas de calor y modificaciones en los patrones de precipitación (periodos de sequías prolongados alternados con periodos de Iluvias intensas) (Parry et al., 2007; Brundland et al., 2012).
En la figura 1 se describe la relación entre el desarrollo económico y el cambio climático, así como las fuerzas motrices que abordan esta problemática a través del desarrollo económico con una visión social, mediante acciones conjuntas entre el Estado, las organizaciones no gubernamentales y la Organización de Naciones Unidas.

Impactos: El impacto ambiental es todo efecto negativo que las actividades humanas producen en el medio ambiente. En la Tabla 2 aparece descrita brevemente la previsión de impactos relacionados con el aumento de temperatura en los sectores identificados como más vulnerables. Con el aumento de temperatura el deshielo, por ejemplo, permite que el permafrost $^{5}$ libere metano, lo que a su vez ha contribuido a favorecer el efecto invernadero. Se han verificado cambios en los sistemas marinos y de agua dulce con el calentamiento global. En el hemisferio Norte se ha comprobado el inicio temprano de la primavera y mayor duración de las estaciones de crecimiento. Estos eventos ya están impactando sistemas agrícolas y de silvicultura (Parry et al., 2007).

Es muy probable que los impactos antes mencionados tengan costos ambientales, que aumenten con el paso del tiempo, por lo que las estimaciones se deben interpretar con cuidado. Por ejemplo, la elevación de la temperatura entre $1-3^{\circ} \mathrm{C}$ prevista para mediados del siglo XXI podrá beneficiar algunos sectores de la agricultura en algunas regiones, condiciones éstas que por otro lado significarán pérdidas en regiones polares y de altitudes. Las regiones y los sectores con elevada exposición a los impactos del cambio climático, que sean más sensibles y / o que tengan menor capacidad adaptativa, serán aquellos para los que los costos netos serán mayores. Es importante destacar que ante un escenario en el que disminuya la emisión de gases de efecto invernadero, se ha evidenciado que sí se podrían evitar algunos impactos adversos del cambio climático (Parry et al., 2007).

Adaptación. La adaptación se refiere al proceso de ajuste de los sistemas naturales y humanos como respuesta a los estímulos climáticos o a sus efectos, mitigando daños o aprovechando circunstancias eventualmente beneficiosas (Parry et al., 2007). También se reconocen como adaptación las políticas, prácticas y proyectos orientados a hacer frente al cambio climático a fin de prevenir daños potenciales. Estas iniciativas pueden ser preventivas o reactivas,

5 Permafrost: capa de hielo congelada, común en el bioma Tundra 
Tabla 1: Gases de efecto invernadero (GEI) que contribuyen al calentamiento global y fuentes principales de emisión. (Fuente: CCAD, 2010; Parry et al., 2007 adaptado)

\begin{tabular}{|c|c|c|c|}
\hline $\begin{array}{l}\text { Gas de efecto invernadero } \\
\text { (GEI) }\end{array}$ & $\begin{array}{l}\text { Concentración } \\
\text { en la atmósfera } \\
\text { (era pre-industrial) }^{1}\end{array}$ & $\begin{array}{c}\text { Concentración } \\
\text { en la atmósfera } \\
(2006)\end{array}$ & Fuentes \\
\hline Dióxido de carbono $\left(\mathrm{CO}_{2}\right)$ & 280 ppm & $379 \pm 0,65$ ppm & $\begin{array}{l}\text { combustión de carburantes } \\
\text { y cambio de uso del suelo }\end{array}$ \\
\hline Metano $\mathrm{CH}_{4}$ & $715 \mathrm{ppb}$ & $1774 \pm 1,8$ & $\begin{array}{l}\text { agricultura y combustión } \\
\text { de carburantes fósiles }\end{array}$ \\
\hline Óxido nitroso $\left(\mathrm{N}_{2} \mathrm{O}\right)$ & $270 \mathrm{ppb}$ & $319 \pm 0,12 \mathrm{ppb}$ & $\begin{array}{l}\text { agricultura: fertilización } \\
\text { nitrogenada }\end{array}$ \\
\hline Clorofluorocarbonos (CFC) & $* *$ & $251 \pm 0,36 \mathrm{ppb}$ & $\begin{array}{l}\text { refrigeración, aerosoles, } \\
\text { espumas y usos industriales }\end{array}$ \\
\hline Hidroclorofluorocarbonos (HCFC) & $* *$ & $169 \pm 1.0 \mathrm{ppb}$ & $\begin{array}{l}\text { Usos en refrigeración, } \\
\text { aerosoles, espumas y } \\
\text { usos industriales }\end{array}$ \\
\hline
\end{tabular}

1 Promedio y rangos naturales; ppm: partes por millón; ppb: partes por billón

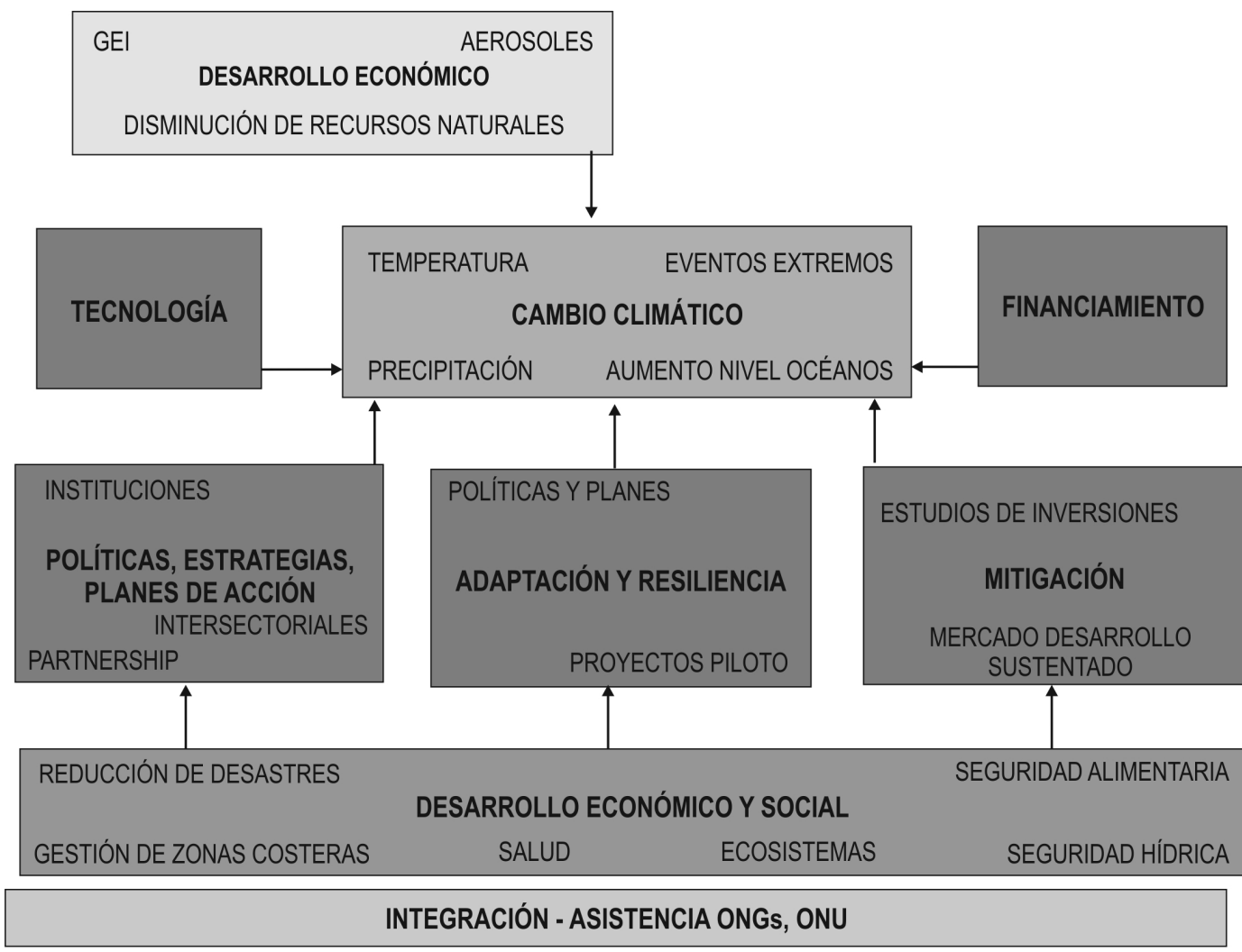

Figura 1. Marco conceptual de las interacciones de las fuerzas motrices antropogénicas del cambio climático (Adaptado: Parry et al., 2007; Côté, 2009; MINAM, 2009). 
Tabla 2. Ejemplos de impactos mundiales previstos para los cambios climáticos asociados a un aumento de temperatura en la superficie mundial, respecto a la temperatura media anual al periodo 1980-1999, para el siglo XXI (Adaptado de Tabla RT3, Parry et al., 2007).

\begin{tabular}{|c|c|}
\hline Sectores & Impactos \\
\hline Agua & $\begin{array}{l}\text { - } \quad \text { Mayor disponibilidad de agua en el trópico húmedo y las latitudes altas. } \\
\text { - } \quad \text { Menor disponibilidad de agua y sequía en aumento en las latitudes medias y bajas. }\end{array}$ \\
\hline Ecosistemas & $\begin{array}{l}\text { Extinción de anfibios y de otras especies sensibles al aumento de temperatura. } \\
\text { Decoloración y mortalidad de corales. } \\
\text {. } \\
\text {. } \\
\text { Inigración de especies. }\end{array}$ \\
\hline $\begin{array}{l}\text { Alimentos \& } \\
\text { agricultura }\end{array}$ & $\begin{array}{l}\text { Inicialmente un aumento en la productividad de los cultivos, pero con el aumento en la } \\
\text { temperatura se disminuirá la producción especialmente de algunos cereales. }\end{array}$ \\
\hline Costas & $\begin{array}{l}\text { - } \quad \text { Aumento de eventos de inundación y tormentas } \\
\text { - } \quad \text { Pérdida de humedales costeros. }\end{array}$ \\
\hline Salud & $\begin{array}{l}\text { - Aumento de malnutrición, enfermedades diarreicas, cardiorrespiratorias, infecciosas. } \\
\text { - } \quad \text { Aumento de morbilidad y mortalidad por olas de calor y sequías }\end{array}$ \\
\hline $\begin{array}{l}\text { Fenómenos } \\
\text { particulares }\end{array}$ & $\begin{array}{l}\text { Retroceso del hielo en Groenlandia y la Antártida occidental. } \\
\text { Reducción de glaciares y aumento de lagos producto del deshielo de glaciares en los } \\
\text { Andes tropicales. }\end{array}$ \\
\hline
\end{tabular}

de carácter privado o público, o surgir de forma autónoma o planificada (MINAM, 2009).

Los sectores incluidos por el IPCC en los procesos de adaptación son la agricultura, el turismo, el ocio, la salud humana, el abastecimiento de agua, la gestión costera, la planificación urbanística y la conservación de la naturaleza (Parry et al., 2007). Las respuestas potenciales de adaptación pueden ser tecnológicas, conductuales, administrativas y/o de políticas públicas.

Existen eventos vinculados al cambio climático como las sequías, las ondas de calor, el retroceso de los glaciares o el aumento de la frecuencia e intensidad de tormentas, que representan nuevos riesgos porque no sabemos cómo enfrentarlos efectivamente y las soluciones dadas inicialmente pueden tener impactos sociales o, aún, llegar a intensificar algunos impactos ambientales no previstos. Por ejemplo, en condiciones extremas de temperatura, los sistemas de climatización la aumentan o disminuyen, haciendo un uso extra de energía, siendo que las prácticas de adaptación recomiendan el consumo controlado de este recurso (Parry et al., 2007). En este sentido, las innovaciones tecnológicas y la industria tienen un enorme reto, al poner en el mercado a precios competitivos, dispositivos climatizadores de ambiente, que usen fuentes alternativas de energía.
Mitigación. Las iniciativas de mitigación tienen un alcance global en el espacio y, a largo plazo, en el tiempo. Estas propuestas son trazadas en consenso durante encuentros periódicos de especialistas representantes de todos los Estados del mundo. Así surgen los denominados protocolos, a los cuales las naciones se pliegan, según su interés.

Dentro de los sectores clave para el proceso de mitigación, el IPCC propuso el energético, el transporte, la industria, el sector residencial, la silvicultura y la agricultura (Metz et al., 2007).

El Protocolo de Kioto $(P K)$ sobre el cambio climático (ONU, 1997) es un instrumento internacional de la Convención Marco de las Naciones Unidas sobre el Cambio Climático (CMNUCC, 1992) que tiene por objeto reducir las emisiones de los GEl durante el período que va desde el año 2008 al 2012, en comparación al promedio histórico de emisiones al año 1990. Se prevé que con la mitigación de los gases de efecto invernadero se reduzca el calentamiento global, con lo que a su vez se podrían evitar algunos impactos adversos.

En el 2005, por segunda ocasión los miembros del CMNUCC se reunieron en Montreal (Canadá) para dar seguimiento al $P K$. Allí se estableció el llamado Grupo de Trabajo Especial sobre los Futuros Compromisos 
de las Partes del Anexo I en el marco del Protocolo de Kioto (GTE-PK), orientado a los acuerdos a tomar después de 2012. La tercera reunión de este grupo se llevó a cabo en Bali, COP13 ${ }^{6}$ (2007), con el foco puesto en las cuestiones Post-2012. La hoja de ruta de Bali llevó al encuentro en Copenhagen (2009), COP $15^{7}$, donde surgió la iniciativa de favorecer la estabilización del clima, con un aumento máximo de $2^{\circ} \mathrm{C}$ sobre la temperatura preindustrial, y un aumento de hasta 450 partes por millón (ppm) de concentraciones de $\mathrm{CO}_{2}$ en la atmósfera. También se propuso la creación de un Fondo Verde y se llegó al compromiso de proporcionar 30.000 millones de dólares en financiación rápida.

En el encuentro celebrado en Cancún en 2010, se decidió aprobar las iniciativas tomadas en Copenhagen 2009, y se aprobó la movilización de $\$ 100.000$ millones de dólares por año a partir de 2020 del Fondo Verde Climático, para atender a las necesidades de los países en desarrollo para combatir el cambio climático, siendo el Banco Mundial la entidad que administre estos recursos. Se acordó también una emisión voluntaria de reducción de emisiones de GEl. Japón, China y Estados Unidos se negaron a firmar cualquier acuerdo vinculante al $P K$. En esta reunión, también se acordó establecer el mecanismo REDD+ en los países en desarrollo.

El sector forestal es responsable por el 17,4\% del total de las emisiones anuales de gases de efecto invernadero por tala y quema de bosques, así como de óxidos de nitrógeno (NOx) provenientes del uso de abonos fertilizantes (UN-REDD, 2008; Stephen, 2009). El mecanismo para la reducción de la deforestación y la degradación forestal para el fomento de las reservas de carbono (REDD+) se vale del sector forestal como mecanismo de mitigación del cambio climático (Angelsen, 2010; Camprubí, 2010). Esta iniciativa de naturaleza financiera ha tenido varias transformaciones: inició en el COP13 (2007) con el objetivo de reducir las emisiones de la deforestación (RED), pasó rápidamente a incluir las emisiones por degradación forestal (REDD), las prácticas de conservación, las prácticas de gestión forestal sostenible y el establecimiento de reservas forestales de carbono. Se presume que sin REDD, el aumento máximo de temperatura en $2^{\circ} \mathrm{C}$ propuesto en Copenhagen no sería alcanzado. (Meri-

COP 13: $13^{\text {a }}$ Sesión de la Conferencia de las partes del de la Convención Marco de Naciones Unidas sobre Cambio Climático, realizada en Bali (2007)

7 COP 15: 15 ${ }^{\text {a }}$ Sesión de la Conferencia de las partes del de la Convención Marco de Naciones Unidas sobre Cambio Climático, realizada en Copenhagen (2009) dian Institut, 2009; Angelsen y Wertz-Kanounnikoff, 2008).

Retomando las iniciativas del Protocolo de Kioto se recuerda el de Mercado de Carbono. El comercio de bonos de carbono es un mecanismo internacional de descontaminación a través de la reducción de emisión de GEl. La idea es que proyectos implementados en países en desarrollo contribuyan a la mejora de la calidad ambiental, regulando la emisión de GEl generada en sus procesos productores. Esta reducción de emisión de $\mathrm{CO}_{2}$ representa un bien canjeable (bonos de carbono), con un precio establecido en el mercado. Así, gobiernos y empresas de distintas partes del mundo compran el derecho a emitir $\mathrm{CO}_{2}$ a través de la compra de estos bonos (MINAM, 2009). Los Bonos de Carbono o Reducciones Certificadas de Emisiones (CERs) constituyen derechos comerciales dentro del mercado de carbono. La reducción de emisión de GEI se mide en toneladas equivalentes de $\mathrm{CO}_{2}$ que se deja de liberar en la atmósfera. Un (1) CER equivale a un bono de carbono, es decir a una tonelada de dióxido de carbono equivalente $\left(\mathrm{eCO}_{2}\right)$. Los CERS pueden ser vendidos a países industrializados, lo que permite mitigar la emisión de GEl, beneficiando a las empresas que no emiten o disminuyen la emisión, y haciendo pagar a aquéllas que emiten más de lo permitido. Las empresas que pueden aplicar a emitir estos certificados son empresas generadoras de energía renovable, de forestación, de limpieza de lagos y de optimización energética de procesos (MINAM, 2009).

El Mecanismo de Desarrollo Limpio (MDL) es un acuerdo también suscrito en el Protocolo de Kioto, que permite que gobiernos de países industrializados inviertan en proyectos de reducción de emisión de GEls en empresas de países en desarrollo o transfieran tecnologías limpias, como una opción para adquirir CERs a menores costos que en el mercado. Los proyectos elegibles para el MDL son de sectores como energía e industria, transporte, residuos y forestal (MINAM, 2009).

El desarrollo sostenible propuesto en Rio-1992 (CNU$M A D, 1992)$ es abordado desde una perspectiva económica y social en la iniciativa Economía Verde (PNUMA, 2011). En este último se propone invertir el dos por ciento del PIB mundial en diez sectores fundamentales de la economía, haciéndolos eficientes en el uso de recursos, disminución de emisiones, sin olvidar a las poblaciones más vulnerables (Tabla 3). En ese sentido el PNUMA (2011) hace un Ilamado a los tomadores de decisiones para que vuelquen su atención a la economía verde, que puede ser aplicada en diferentes niveles de gestión (UN, 2012). 
En el Foro para el desarrollo sostenible Estocolmo +40, realizado en abril de 2012 se dieron recomendaciones de cómo gobiernos, economías, organizaciones e individuos pueden promover y contribuir con este importante proceso.

En Rio +20 , la Conferencia de la Naciones Unidas sobre Desarrollo Sostenible, a celebrarse en junio de 2012 se tratarán temas como el comercio y economía verde, migración, dinámica poblacional, transporte bajo en carbono en economías emergentes, ciencia y tecnología, agua, gobernanza, seguridad alimentar y agricultura, reducción de riesgos, empleos verdes e inclusión social, ciudades, océanos, todos ellos entorno al desarrollo sostenible. En este encuentro se espera asegurar un compromiso político con el desarrollo sostenible a pesar del escepticismo generado por la crisis económica mundial.

Vulnerabilidad. Es el grado de susceptibilidad o incapacidad de un sistema para afrontar los efectos negativos del cambio climático, en función del índice de variación climática, su sensibilidad y su capacidad de adaptación. Para ser más objetivos, la vulnerabilidad se determina a escala regional. También se considera vulnerabilidad al riesgo de impacto negativo del cambio climático en la naturaleza y en los seres humanos, que está implícito en la variabilidad (MINAM, 2009).

La evaluación de las vulnerabilidades clave ayudará a los tomadores de decisión a proponer las acciones convenientes frente a los riesgos del cambio climático para una región. Los criterios más usados para establecer si un evento se puede definir como vulnerabilidades clave son su magnitud, periodicidad, persistencia y/o reversibilidad, su potencial de adaptación, los aspectos de la distribución, las probabilidades e importancia de sus impactos, entre otros. Las vulnerabilidades clave están presentes en los sistemas biológicos, geofísicos, sociales y económicos, siendo que en los dos primeros hay implícita una menor capacidad de adaptación que en los dos últimos. Existen factores identificados como amplificadores de la vulnerabilidad, que están también presentes a nivel regional en Latinoamérica, como son el crecimiento urbano desordenado, el desplazamiento y migración por conflictos por los recursos y la crisis estructural del sector rural y del agro (MINAM, 2009).

La vulnerabilidad futura obedece estrechamente a las vías de desarrollo, siendo que ya fue evidenciado que regiones más pobres son más sensibles. La pobreza, el acceso desigual a los recursos, la inseguridad alimentaria, la globalización económica, los conflictos, las enfermedades, intensifican estos procesos y eventos, así como la contaminación marina, las escorrentías de agroquímicos, la acidificación del océano.

En septiembre del año 2000 tuvo lugar en Nueva York la Cumbre del Milenio, en la cual representantes de 189 naciones firmaron la Declaración del Milenio, en la que se plantean los ocho objetivos sobre la erradicación de la pobreza. Los objetivos son: erradicar la pobreza extrema, lograr la enseñanza primaria universal, promover la igualdad entre los géneros, reducir la mortalidad infantil, mejorar la salud materna, combatir el HIV-Sida y otras enfermedades, y garantizar la sostenibilidad del medio ambiente, así como el fomentar la asociación mundial para el desarrollo, siendo que esta última herramienta garantiza económicamente la consecución de los siete primeros objetivos para el año 2015. Cada objetivo se evaluará a través de 18 metas, con 48 indicadores (ONU, 2000; ONU, 2008). Es probable que el cambio climático haga más difícil el desarrollo sostenible y, por ende, los objetivos de Desarrollo del Milenio, a través de una mayor exposición a los impactos adversos o indirectamente mediante el desgaste de la adaptación (MINAM, 2009, PNUD, 2009; ONU, 2000)

Adaptación y mitigación. Es importante mencionar que los programas de adaptación y de mitigación van de la mano, porque tienen un alcance temporal, espacial e institucional diferente pero complementario. La mitigación tendrá beneficios a escala mundial, que sólo serán percibidos a mediados del S. XXI. Su ejecución es necesaria para disminuir la magnitud de los efectos del cambio climático. De otro lado, los programas de adaptación tienen un alcance regional y pueden ser inmediatos si abordan también las vulnerabilidades a las condiciones climáticas presentes. Así, a largo plazo, una combinación de medidas de mitigación, de adaptación y de investigación en ciencia básica e innovación sería la combinación que más se ajustaría a los procesos de gestión del cambio climático. La búsqueda de la combinación ideal resulta equivocada dado que es difícil captar los intereses individuales de todos los sectores afectados. Sin embargo, la vía del desarrollo sostenible es un mecanismo para promover tanto la adaptación como la mitigación (Parry et al., 2007; Field et al., 2009).

La mitigación está fundamentada en acuerdos internacionales y en la aplicación de políticas públicas. Para su avance, se requiere de la participación de los grandes emisores de gases de efecto invernadero a nivel mundial. Los procesos de adaptación tienen lugar a niveles nacional y regional, motivados por actores privados de las entidades afectadas y por acuerdos de carácter público alcanzados por las comunidades 
afectadas. Existen ejemplificaciones de sinergias positivas entre los procesos de adaptación y mitigación como son los programas de electrificación rural basada en fuentes de energía renovables, la arborización urbana, el desarrollo de sistemas agroforestales, así como la forestación de laderas que, además de retener el carbono, controla la erosión. Estas sinergias pueden aumentar la relación costo-eficiencia de las acciones, pero no siempre son implementadas por la falta de información conceptual y empírica que dificulta la evaluación de las necesidades y el potencial de las sinergias considerando explícitamente la adaptación y la mitigación (Parry et al., 2007; PNUMA, 2011).

\section{Inquietudes del proceso}

La capacidad adaptativa no es igual entre ni dentro de los grupos sociales, ya que depende de las condiciones económicas y naturales, de las redes sociales, de las instituciones públicas, de las legislación y del cumplimiento de las leyes, y hasta de los tratados de libre comercio. De esta manera, en los países en desarrollo los recursos económicos son desviados para suplir otras necesidades diferentes a la adaptación al cambio climático.

Considerando que el cambio climático está originado principalmente en la elevada emisión de $\mathrm{CO}_{2}$ por el uso de combustibles fósiles, los la producción de biocombustibles se han convertido en un nuevo paradigma principalmente de los países tropicales (Rodríguez, 2008), en donde el cultivo de alimentos dejó de tener como destino natural las plazas de mercado y la alimentación de la población, para dirigirse hacia las producción de combustibles. La búsqueda de desarrollo económico es la justificativa para los cambios en el uso del suelo. Extensas áreas de bosque natural son reemplazadas por monocultivos de caña de azúcar y de palma de aceite, alterando el curso de cuerpos de agua, favoreciendo así el empobrecimiento y desplazamiento de comunidades rurales. Sin embargo, en la región existen experiencias valiosas. Costa Rica se ha consolidado como un país conservacionista de sus recursos naturales y ha canalizado su desarrollo económico en esa dirección. La calidad del producto turístico que combina paisaje natural y seguridad, han colocado a este país latinoamericano en referencia sobre programas de conservación y la biodiversidad.

De otro lado, la implementación de programas de comercio de bonos de carbono, a través de CERs o de las diferentes modalidades de REDD requiere de la supervisión y apoyo de los gobiernos regionales y nacionales, para garantizar que los importantes recursos económicos que surjan de estas iniciativas lleguen a las comunidades locales. A pesar de que el programa REDD pretende reducir significativamente la emisión de GEI de forma rápida respecto a otras alternativas de mitigación y es comparativamente barata, su puesta en marcha se vuelve realmente compleja, ya que los mecanismos de monitoreo, verificación y equidad (Angelsen, 2008) todavía están en discusión. La participación de las comunidades indígenas, campesinas y locales en el mecanismo y acciones REDD va a marcar la diferencia en los resultados de implementación de esta iniciativa. Los riesgos y oportunidades que el programa REDD puede significar para las comunidades menos favorecidas incluyen, de una parte, la pérdida potencial de acceso a la tierra y, de otro lado, un eventual incremento de recursos económicos que lleguen a áreas rurales pobres y que mejoren la gobernabilidad del bosque. En ese sentido, la participación de las comunidades en la implementación de REDD aumenta la probabilidad de que sus riesgos se mitiguen y de que las oportunidades aumenten.

\section{Oportunidades de gestión ante el cambio climático. Nivel regional, nacional y local}

El impacto del cambio climático en América Latina será evidente en diferentes ejes: se pronostica una posible extinción de las especies de la sabana central brasilera (sabanización), la pérdida de las especies forestales y de biodiversidad de la Amazonía, así como el declive de la productividad de los ecosistemas. Se prevé también la desaparición de glaciares tropicales de latitudes medias y una disminución general de la disponibilidad de hídrica (Magrin et al., 2007). Otros acontecimientos ya percibidos son la menor duración de la temporada de lluvias: eventos muy intensos alternados con falta de precipitación, aumento del frío nocturno y del calor durante el día en las tierras altas (heladas), poniendo en riesgo la seguridad alimentaria de los pequeños productores (PNUD, 2009).

El cambio climático ha favorecido en los Andes Tropicales transformaciones ecológicas como desplazamientos de pisos ecológicos, con modificaciones de los regímenes hídricos (Postigo, 2009). En estudios realizados en los Andes peruanos, se ha verificado que el cambio climático ha impactado negativamente la infraestructura de las familias campesinas dedicadas a la agricultura y al pastoreo de subsistencia. Es importante mencionar que el IPCC resalta la escasa calidad y cantidad de información originada en la región, por lo que se intuye que hay una serie de impactos regionales aún sin considerar (Magrin et al., 2007).

Para la elaboración de una estrategia regional de gestión frente al cambio climático, es necesario conocer 
Tabla 3: Principales sectores a invertir en economía verde. (Adaptado de PNUMA, 2011)

\begin{tabular}{|c|c|c|}
\hline Sector & Activos y Retos & Enverdecimiento del sector \\
\hline $\begin{array}{l}\text { Diversidad } \\
\text { biológica }\end{array}$ & $\begin{array}{l}\text { Servicios de ecosistemas: Genes, especies, ecosistemas, } \\
\text { bosques, cuerpos y corrientes de agua, silvicultura, garantizar la } \\
\text { estabilidad del ciclo hídrico. } \\
\text { Recreación, Regulación hídrica, almacenamiento de carbono, } \\
\text { alimentos, fibras, combustible, polinización, avances médicos, } \\
\text { capacidad de adaptación. Mediante la promoción y difusión } \\
\text { de prácticas sostenibles, se facilita acceso a los alimentos a las } \\
\text { personas más desfavorecidas, se promueve la reducción de la } \\
\text { pobreza, se incrementa el secuestro de carbono y facilita el } \\
\text { acceso a los mercados internacionales de los productos verdes. }\end{array}$ & $\begin{array}{l}\text { Agua y sus beneficios para la agricultura y los } \\
\text { hogares, protección de cuencas hídricas } \\
\text { el ciclo del carbono y su función en la } \\
\text { mitigación del clima, los microclimas locales } \\
\text { respecto a la seguridad del hábitat, }\end{array}$ \\
\hline Agricultura & $\begin{array}{l}\text { Aplicación de prácticas de cultivo racionales como el uso } \\
\text { eficiente del agua, la utilización extensiva de nutrientes } \\
\text { orgánicos y naturales para el suelo. Preparación optima de los } \\
\text { cultivos y el control integrado de plagas. }\end{array}$ & Alimentar 9000 millones de personas al 2050 \\
\hline Agua & $\begin{array}{l}\text { Garantiza la estabilidad del ciclo hídrico. } \\
\text { Seguridad hídrica de personas y ecosistemas en términos de } \\
\text { escasez, sobreabundancia (riesgo de inundación) y calidad. } \\
\text { Cuando los servicios de saneamiento no son los adecuados, } \\
\text { las enfermedades transmitidas por el agua suponen un gasto } \\
\text { elevado. }\end{array}$ & $\begin{array}{l}\text { Mejora los acuerdos institucionales y los } \\
\text { sistemas de concesión de derechos y de } \\
\text { asignaciones, ampliar la aplicación de cobros } \\
\text { por los servicios de los ecosistemas, reducir } \\
\text { los subsidios a la inversión y mejorar el cobro } \\
\text { por agua y los acuerdos financieros. }\end{array}$ \\
\hline Pesca & $\begin{array}{l}\text { Las reservas pesqueras de proteínas. } \\
\text { Los subsidios han provocado un exceso de capacidad dos veces } \\
\text { superior a la capacidad reproductiva de los peces }\end{array}$ & $\begin{array}{l}\text { Desarrollo económico, el empleo, la } \\
\text { seguridad alimentaria y el sustento de } \\
\text { millones de personas en el mundo }\end{array}$ \\
\hline $\begin{array}{l}\text { Energía } \\
\text { renovables }\end{array}$ & $\begin{array}{l}\text { Facilitar energía a los } 1.600 \text { millones de personas que no tienen } \\
\text { acceso actualmente a la electricidad. Mitigar la pobreza y } \\
\text { mejorar la calidad de vida. Mejorar el nivel de vida y la salud } \\
\text { en las áreas de ingresos bajos, especialmente en las zonas más } \\
\text { aisladas. }\end{array}$ & $\begin{array}{l}\text { Entre las soluciones más rentables se } \\
\text { encuentran la biomasa limpia y la energía } \\
\text { solar fotovoltaica no conectada a la red, } \\
\text { que ofrecen costos de operación reducidos } \\
\text { y flexibles y la posibilidad de utilizarlas a } \\
\text { pequeña escala }\end{array}$ \\
\hline $\begin{array}{l}\text { Turismo } \\
\text { planificado }\end{array}$ & $\begin{array}{l}\text { Favorece el desarrollo económico. Hay emisión de gases de } \\
\text { efecto invernadero, consumo de agua, vertimientos de agua no } \\
\text { tratada, generación de residuos, danos a la biodiversidad terrestre } \\
\text { y marina o amenaza para la supervivencia de la cultura y las } \\
\text { tradiciones locales }\end{array}$ & $\begin{array}{l}\text { La participación de la comunidad local, } \\
\text { especialmente de los mas pobres, en } \\
\text { la cadena de valor del turismo resulta } \\
\text { fundamental para desarrollar la economía } \\
\text { local y reducir la pobreza }\end{array}$ \\
\hline Edificaciones & Ecoeficientes. & $\begin{array}{l}\text { Aumentar la eficiencia energética y de } \\
\text { consumo de agua }\end{array}$ \\
\hline Transporte & Alternativas no emisoras de GEI. & Aumentar la eficiencia energética y emisiones. \\
\hline Residuos & $\begin{array}{l}\text { Reducción, Reciclar, Reutilizar. Producción de abono. } \\
\text { Producción de energía a partir de biogás }\end{array}$ & $\begin{array}{l}\text { Aumentar su aprovechamiento; disminuir su } \\
\text { vertimiento en Rellenos Sanitarios }\end{array}$ \\
\hline Industria & Reducción de emisiones y de consumo de energía. & $\begin{array}{l}\text { Aumentar la eficiencia energética y emisiones. } \\
\text { Generación de empleo en estas áreas. }\end{array}$ \\
\hline
\end{tabular}

las políticas y el plan de desarrollo nacional. En ese contexto, a través de estudios integrados de vulnerabilidad y adaptación, se identifican zonas y sectores vulnerables, con los que se planean proyectos de adaptación. La adopción de programas de energías renovables y de eficiencia energética en los diversos sectores productivos son oportunos (Paz-Cigarán y García, 2006).
En el ámbito regional, los gobiernos, la sociedad civil, los grupos empresariales y los centros de investigación se basan en lo anterior para establecer sus prioridades, planificar el apoyo y las medidas que permitan reducir los factores de vulnerabilidad existentes. En un ámbito menor, las comunidades locales, ONGs, los gobiernos locales y los grupos empresariales deben unirse con los sectores vulnerables y participar 
conjuntamente para identificar qué grupos y qué factores constituyen amenazas mayores y cómo reducir su nivel de vulnerabilidad.

A continuación se describirán algunas experiencias regionales de gestión ambiental en el contexto del cambio climático:

\section{Caso 1: Estado de Sao Paulo, Brasil.}

El Gobierno del Estado de Sao Paulo, Brasil, está llevando a cabo algunas iniciativas de desarrollo sostenible, que cobijan diferentes sectores. La federación de industrias de este estado (FIESP) se propone reducir las emisiones de $\mathrm{CO}_{2}$, así como apoyar proyectos de investigación en energías renovables, en asocio con la Escuela Politécnica de la Universidad de Sao Paulo.

De igual forma, el Estado pretende ampliar la extensión de parques estatales, con lo que el Jardín Botánico de Sao Paulo aumentará su área de Bosque Atlántico nativo, colaborando así con la preservación de este bioma.

En el área de la gestión ambiental, una resolución conjunta de las Secretarias del Medio Ambiente y de Hacienda establecerá un grupo de trabajo que tendrá como objetivo estudiar las adecuaciones en la legislación del Estado de Sao Paulo que posibiliten la implementación integral de las políticas estatales ambientales, como la Política Estatal del Cambio Climático y el Plan de Control de la Contaminación Vehicular. También, la Estrategia para el Desarrollo Sustentable del Estado de Sao Paulo 2020 pretende establecer metas sectoriales para definir la acción del gobierno del Estado hasta el año 2020.

De otro lado, han sido firmados nuevos términos de compromiso de responsabilidad post-consumo. La idea es que fabricantes e importadores de productos y empaques presenten una propuesta de implantación del Programa de Responsabilidad Pos-consumo que indique un conjunto de acciones, procedimientos y medios destinados a viabilizar la colecta y la restitución de los residuos sólidos para el reaprovechamiento en su ciclo o en otro ciclo productivo, o en otro destino final ambientalmente adecuado, inicialmente en los sectores de operadoras de telefonía celular, de llantas, de aceites lubricantes y de aceites combustibles (SMA, 2012).

\section{Caso 2: Comunidades campesinas de los Andes Peruanos}

En Perú, la estrategia nacional de cambio climático se focaliza en la diversidad biológica y en la deser- tificación, con 11 líneas estratégicas nacionales para el cambio climático y una agenda de investigación científica nacional en cambio climático (MINAM, 2009).

En los departamentos de Puno, Arequipa y Cuzco se desarrollaron acercamientos con los actores de las tierras altas para conocer sus necesidades y establecer programas de inversión en las áreas más sensibles en el contexto del cambio climático (Postigo, 2009). Dentro de las prioridades se observó:

1. Uso del agua: el uso del agua, además de ser productivo (para riego, consumo humano, generación de energía), debe incluir el papel ecológico que ella desempeña en la formación del suelo, el mantenimiento de una flora de microorganismos que garanticen la productividad agrícola y ganadero. Establecer un esquema de uso del agua por parte de los pobladores de las tierras altas y bajas, para evitar tensiones entre estos grupos, entre la economía de mercado y la de subsistencia, y entre la cultura agrícola y la pastoril.

2. Uso del suelo: la expansión de la franja de cultivos a tierras más altas, ha convertido pastizales en parcelas, destruyendo sumideros de carbono muy antiguos, por lo que es evidente el desconocimiento de los pastos como ecosistemas que secuestran carbono.

En otra aproximación a comunidades altoandinas de las microcuencas Challhuahuacho (Apurima) y Santo Tomás (Cusco), también en Perú, cuatro organismos internacionales PNUMA, PNUD, OPS y FAO se unen para desarrollar el programa en el Marco de Asistencia al Desarrollo de las Naciones Unidas (MANUD), para promover el acceso a los servicios sociales básicos, apoyar y promover el desarrollo de oportunidades económicas, fortalecer las capacidades del Estado y para prevenir y manejar situaciones de riesgo social, sanitario y desastres naturales en esta región. (PNUD, 2008).

\section{Caso 3: Colombia}

Los impactos proyectados del cambio climático en Colombia se enmarcan en los ya listados para América Latina anteriormente (Marin, 2009): cambios en los regímenes hidrológicos con reducción en los escenarios más pesimistas, de entre un 15\% hasta de un $36 \%$ en las precipitaciones (Ruiz, 2010), lo que incrementará el déficit hídrico en cuencas abas- 
tecedoras de acueductos, embalses hidroeléctricos y distritos de riego. Los departamentos más afectados serían los de la Costa Caribe, como Bolívar, Sucre, Córdoba, Magdalena, Atlántico, principalmente hacia finales del S. XXI, cuando se espera que el clima pase de semi-húmedo (condiciones actuales) a semiárido y posteriormente a árido. En la Región Andina se prevé que el clima vaya de semi-húmedo a semi-árido en los departamentos de Cundinamarca, Boyacá, Tolima, Huila y el oriente del Valle, principalmente. La humedad relativa se reducirá entre $1.8 \%$ en la primera mitad hasta 5\% hacia 2100.

De acuerdo a modelos climáticos más apurados, se prevé un aumento en la temperatura media de $1,4^{\circ} \mathrm{C}$ hasta 2040 , de $2,4^{\circ} \mathrm{C}$ hasta 2070 y de $3,2^{\circ} \mathrm{C}$ hasta 2100 , con respecto al periodo de referencia 1971 2000, siendo las regiones más afectadas la Caribe y Andina, especialmente los departamentos de Sucre, Norte de Santander, Risaralda, Huila y Tolima (Ruiz, 2010). Se observarán deslizamientos, inundaciones, vendavales, olas de calor y sequías, lo que conllevará a un aumento en el número de personas afectadas, así como desplazamientos ecológicos en las regiones de alta montaña. Esto llevará a una disminución y afectación de los bienes y servicios ambientales de todos los ecosistemas, lo que generará conflicto por el acceso y uso de los recursos naturales (Marín, 2009).

De continuar el cambio observado, con una tasa de pérdida de los glaciares de 3 a $5 \%$ de su área, éstos podrían desaparecer en 40 años. El nivel del mar Caribe subiría entre 23 y $32 \mathrm{cms}$ a final del siglo XXI y el océano Pacífico hasta $21 \mathrm{cms}$ en ese mismo periodo (Ruiz, 2010).

Colombia cuenta con un desarrollo institucional y jurídico en el campo ambiental, especialmente desde la reforma constitucional de 1991 (Cardona, 2009). El Ministerio de Ambiente y Desarrollo Sostenible es el rector de la política ambiental en Colombia.

El primer proyecto de adaptación al cambio climático en Colombia se inició en el año 2000 y para el 2009 se contaban con más de 40 iniciativas que congregan más de 100 instituciones.

En el año 2002, se establecieron los lineamientos de una política para el cambio climático en este país, con seis estrategias principales:

1. Mejorar la capacidad de adaptación a los impactos del cambio climático

2. Promover la reducción de emisiones por fuentes y absorción por sumideros de GEI
3. Disminuir los impactos de las medidas del Protocolo de Kioto sobre las exportaciones de combustibles fósiles

4. Promover la investigación y fortalecer el sistema de información en cambio climático

5. Promover la divulgación y concientización pública

6. Promover mecanismos financieros para el desarrollo de las estrategias y líneas de acción de esta política (MMA, DNP, 2002)

En el contexto de la mitigación, Colombia es el cuarto país de Latinoamérica en número de proyectos MDL registrados y onceavo en el mundo (MAVDT, 2011). Para el segundo semestre de 2010, Colombia contaba con 161 proyectos en MDL, con un potencial anual de reducción de emisiones de GEl de 21.743.499 Ton $\mathrm{eCO}_{2} / \mathrm{año}$. De estos proyectos, 9 emiten CERs con un potencial total de ingresos por CERs de proyectos del portafolio por cerca de US\$190.000.000 de dólares al año. De estos proyectos, el sector industrial tiene el $37,89 \%$, seguido del sector energético con un $24,84 \%$, residuos con $17,39 \%$, forestal con $11,8 \%$ y transporte con 8,07\% (MAVDT, 2011).

Actualmente, se han emitido CERs para nueve de estos proyectos, principalmente del sector energético (energía renovable (5), industrial (substitución de combustible y cambio de hornos, y reducción de NOx), transporte (fase II-IV de Transmilenio) y residuos (relleno sanitario)) (MAVDT, 2011).

Dentro de las acciones regionales, en su momento el MAVDT (Ministerio de Ambiente, Vivienda y Desarrollo Territorial) y el Instituto de Hidrología, Meteorología y Estudios Ambientales (IDEAM), crearon una página web (http://www.cambioclimatico.gov.co) con información proveniente de diferentes sectores gubernamentales, de investigación, académico y de ONGs para la formación y fortalecimiento de las capacidades en temas del cambio climático en el país.

\section{Caso 4: La Orinoquía colombiana y sus perspectivas de desarrollo}

La región de la cuenca del Río Orinoco está ubicada en el sur oriente colombiano, limitando con la Amazonía al sur. La Orinoquía constituye el 30.4\% del territorio nacional colombiano, incluyendo la totalidad de los departamentos de Vichada, Arauca y Casanare, así como el $95 \%$ del departamento del Meta, y una menor proporción de los departamentos de Caquetá, Cundinamarca, Guainía, Guaviare, Huila, Norte 
de Santander, Santander y Vaupés (Rodríguez et al., 2009; Correa et al., 2006). La región posee el $32 \%$ de las existencias de agua dulce del país y $38 \%$ de las micro-cuencas.

La Orinoquía está conformada por humedales, selvas húmedas, ecosistemas de montaña y sabanas tropicales, siendo estas últimas el paisaje por el que la región es principalmente reconocida. De esta heterogeneidad de ecosistemas (alrededor de 156), 92 son reconocidos como naturales (Romero et al., 2004). La región ha sido catalogada como una de las áreas silvestres del planeta, por su riqueza ornitológica (McNish, 2007), por poseer una de las mayores riquezas acuícolas del mundo (Maldonado-Ocampo et al., 2008; IAvH 2009), por su riqueza en gramíneas tropicales, anfibios, reptiles, mamíferos, entre otros. Así, este territorio es considerado como un lugar donde coincide la alta biodiversidad y al mismo tiempo una alta amenaza por pérdida (Mittermeir et al., 1994; Correa et al., 2006).

Con el cambio climático se espera que la temperatura aumente $2,7^{\circ} \mathrm{C}$ para el año 2050 , con reducción en la precipitación entre $10 \%$ y $20 \%$ como consecuencia del cambio climático (IDEAM, 2007). Estas condiciones favorecen los procesos de desertificación de las sabanas y del bosque basal, alterando los procesos de agricultura y ganadería que en la actualidad de desarrollan, profundizando los conflictos por el acceso al agua durante la época seca. De otro lado, se prevé un aumento en las precipitaciones en Vichada, Vaupés y Guainía (Ruiz, 2010).

Las diversas formas de ver la Orinoquía están representadas en sus actores. El Estado considera la Orinoquía como un territorio sinónimo de oportunidades que debe ser "explorado y colonizado" (Arias, 2004). El Departamento Nacional de Planeación (DNP, 2007) propone la agroindustria, los proyectos agropecuarios y el desarrollo de proyectos forestales a gran escala como opciones para acelerar el desarrollo de la región, involucrando al sector privado, nacional e internacional, sin que sea claro el papel de los gobiernos locales, de las diferentes organizaciones ni asociaciones civiles y privadas, con lo que es factible la exclusión de campesinos y productores, favoreciendo un crecimiento desordenado de las ciudades y sus consecuentes cambios demográficos (Rodríguez et al., 2009).

Con las tasas de crecimiento económico mundial, la demanda creciente por combustibles es evidente. La Orinoquía es conocida y reconocida por la explotación de hidrocarburos en Colombia (Viloria, 2009). En la región el departamento de Arauca lideró la producción de petróleo crudo con la 209395 bpdc (ba- rriles por día calendario) en 1990, superado 9 años después por Casanare con 475138 bpdc. Este liderazgo fue asumido por el Meta una década después con una producción de 335013 bpdc, equivalentes al $61,2 \%$ de la producción de hidrocarburos pesados de la región (Minminas, 2011). Sin embargo, el contexto del cambio climático exige la búsqueda de combustibles alternativos que no favorezcan el calentamiento global. Es así como la producción de biocombustibles, principalmente para exportación, es una opción de adaptación que ha tomado fuerza en la región.

Como parte del paquete de desarrollo agropecuario de la Orinoquía, el cultivo de palma de aceite para producción de biodiesel, está apoyado a través de una serie de subsidios que permitan aumentar la producción y exportaciones de aceite de palma, proponiéndose pasar de 500.000 toneladas en Colombia en 1999 a cerca de 3,5 millones de Ton en el 2020 (FEDEPALMA, 2007). A pesar del reconocimiento por parte del capital privado de la importancia del establecer de modelos sostenibles de desarrollo con compromiso ambiental y social, la especulación ocasionada por la invasión de capitales que buscan comprar tierras para dedicarlas a la producción agrícola a gran escala, podría ocasionar el deterioro de ecosistemas naturales como consecuencia del drenaje de suelos para el cambio de su uso, lo que puede también alterar otros ecosistemas naturales.

En general, la riqueza en biodiversidad se percibe negativa en relación con las posibilidades de desarrollo económico al considerarse que la principal función de la biodiversidad es la de ser fundamento de la dinámica y de la productividad de los ecosistemas, ya sean estos naturales, semi-naturales ${ }^{8} \mathrm{o}$ transformados (Rodríguez et al., 2009). Los servicios ambientales surgen de la relación dinámica hombre-clima-aguaecosistemas. En los servicios de regulación, principalmente del agua, interactúan diversos ecosistemas que soportan el ciclo hídrico (EEM, 2004), que para la Orinoquía se han identificado como páramos, selvas andinas, bosques de niebla, sabanas y superficies inundables, humedales y ríos. La disponibilidad del agua en esta región está sujeta a ciclos estacionales, por lo que cualquier alteración va a repercutir en la dinámica de su sistema.

\section{Conclusiones}

Las acciones de mitigación, adaptación e identificación de las vulnerabilidades de cada región en par-

8 En los ecosistemas seminaturales su estructura y función son resultado de la acción humana (Rodríguez et al. 2009). 
ticular son procesos en evolución constante y de características dialécticas porque involucran la interacción de todos sus actores y elementos. Estos procesos dependen de la voluntad política, económica y de la inversión en estudios en tecnología, ciencia básica y ciencias sociales, que nos dan las luces indispensables para identificar opciones alternativas a las adoptadas hasta ahora.

El costo del cambio climático ya alcanzó pérdidas abrumadoras no sólo en bienes materiales, sino en biodiversidad y vidas humanas, que los economistas calculan en billones de dólares, independientemente de que la vida no pueda tasarse en dinero. Sabemos que podremos adaptarnos a sus consecuencias, pero ¿Podremos pagarlo? ¿Podremos asumir los costos de la construcción de diques en las ciudades costeras, el costo de las evacuaciones y re-ubicación de las comunidades pobres, desplazadas por eventos naturales como deslizamientos o inundaciones?

Nuevas aproximaciones económicas sobre inversiones "verdes" permiten predecir que, a largo plazo, la economía verde mejora el rendimiento económico y puede incrementar la riqueza mundial, a la vez que se recuperarán las reservas de recursos renovables, se reducirán los riesgos ambientales y se reconstruirá nuestra capacidad para generar prosperidad en el futuro.

Durante la década pasada (2001-2010) se generaron cambios dramáticos en el paradigma económico mundial, poniendo de manifiesto deficiencias estructurales y de riesgo que aún no se han resuelto. Gran parte del capital se invirtió en propiedades, combustibles fósiles y activos financieros, lo que resultó en una crisis económica y social a nivel mundial. Poco capital fue invertido en conocimiento de energías renovables, de eficiencia energética, transporte público, agricultura sostenible, protección de los ecosistemas o de la diversidad biológica, lo que pone de manifiesto un modelo de desarrollo inmediatista.

Como especie humana tenemos una importante capacidad de adaptación que nos permite responder a peligros inminentes, aunque es evidente que esa capacidad no nos garantiza comprender las consecuencias presentes y futuras del cambio climático. Existen evidencias contundentes de que el enverdecimiento de la economía no disminuye la creación de riqueza ni de oportunidades, invalidando así la contradicción falsa que opone la sostenibilidad ambiental y el crecimiento económico. Antes bien, un pacto inteligente entre estos dos es lo que ha de derivar en progreso social. Como sociedad, es fundamental priorizar el bienestar común de las generaciones presentes y futuras.

\section{Referencias}

Angelsen A. Policies for reduced deforestation and their impact on agricultural production PNAS, 2010; 107(46): 19639-19644.

Angelsen A, Wertz-Kanounnikoff S. 2008. What are the key design issues for REDD and the criteria for assessing options? En Angelsen A. (Editor). Moving Ahead with REDD: Issues, Options and Implications. Center for International Forestry Research (CIFOR). Bogor, Indonesia.

Angelsen A. (Editor). 2008. Moving Ahead with REDD: Issues, Options and Implications. Center for International Forestry Research (CIFOR). Bogor, Indonesia.

Arias J. 2004. Ganadería, paisaje, territorio y región: una historia ecológica y social de la Orinoquia colombiana. Instituto de Investigación de Recursos Biológicos Alexander von Humboldt. Programa, uso y valoración. Línea saberes locales y uso de biodiversidad. Bogotá.

Brundland G, Ehrlich P, Goldemberg J et al. Environment and development challenges: the imperative to act. Blue Planet Synthesis paper for UNEP. London. 2012; [fecha de acceso (12-06-2012)] URL: http://www.unep.org/pdf/pressreleases/ Blue_Planet_synthesis_paper.pdf

Callendar GS. The artificial production of carbon dioxide and its influence on temperature. Quarterly Journal of the Royal Meteorological Society, 1938;(64): 223-237.

Camprubí A. 2010. Mecanismo para la reducción de la deforestación y la degradación forestal y para el fomento de las reservas de carbono (REDD+): Nota sobre un innovador instrumento de mitigación. Revista Catalana de Dret AmbientaL, I (2): 1 - 24

Cardona A. 2009. Actores relacionados con el abordaje del Cambio climático en Colombia. Mapeo institucional. PNUD.

Correa H, Ruiz S, Arévalo L (Editores). 2006. Plan de acción en biodiversidad de la cuenca del Orinoco-Colombia, 20052015. Propuesta técnica. Corporinoquia-Cormacarena$\mathrm{IAvH}$ - Unitrópico - Fundación Omacha-Fundación Horizonte Verde-Universidad Javeriana-Unillanos-WWF Colombia-GTZ Colombia. Bogotá.

Côté M. Estrategias de la Naciones Unidas para responder al cambio climático. PNUD. 2009; [fecha de acceso: 0110-2011] URL: http://www.pnud.org.co/img_upload/ 61 626461626434343535373737353535/CAMBIOCLIMATICO/1.\%20Productos $\% 20$ del\%20Proyecto $\% 20$ de $\% 20$ Transversalizaci\%C3\%B 3n\%20del\%20Cambio\%20 Clim\%C3\%A1tico/1.1\%20Pres\%20PW\%20Point\%20Curso $\% 20 C C / 5 . \% 20$ Estrategia $\% 20$ NU\%20frente\%20CC. $\% 20$ Marjolaine\%20PNUD.pdf

Crowley T, North G. 1988. Abrupt Climate Change and Extinction Events in Earth History. Science, 240 (4855): 996-1002

CCAD. SICA - Comisión centroamericana de Ambiente y DesarroIlo. Sistema de la Integración Centroamericana. 2010. Estrategia Regional de cambio climático. 
CMNUCC- Conversión Marco de la Naciones Unidas sobre Cambio Climático. 1992; [fecha de acceso 01-10-2011] URL: http://unfccc.int/portal_espanol/essential_background/ feeling_the_heat/items/3385.php

CNUMAD - Conferencia de las Naciones Unidas para el Medio Ambiente y Desarrollo de Río de Janeiro, Brasil. 1992; [fecha de acceso 01-10-2011] URL: http://www.un.org/spanish/conferences/wssd/unced.html

EEM- Evaluación de los ecosistemas del milenio (MEA, millenium ecossistem assesment, en inglés), 2004; [fecha de acceso 0110-2011] URL: http://www.millenniumassessment.org

Field C, Barros V, Ebi K. 2009. Working Group 2. Findings from AR4, expectations from AR5. Impacts, Adaptation and Vulnerability. Contribution of Working Group II. IPCC Secretariat, Geneve, Switzerland, p. 8 .

FEDEPALMA - Federación Nacional de Cultivadores de Aceite de Palma. 2007. Gestión ambiental del sector palmero en Colombia frente al desarrollo sostenible. En Quevedo L, Baquero O. (Editores). Memorias. Una década de gestión ambiental corporativa en la Orinoquia colombiana 1995-2005. Corporación Autónoma Regional de la Orinoquia. Yopal. Colombia.

IDEAM - Instituto de hidrología, meteorología y Estudios Ambientales. INAP - Instituto Nacional de Administración Pública. 2007; [Acceso 01-10-2011] URL: http://www.cambioclimatico.gov.co/jsp/loader.jsf?|Servicio=Publicaciones\& $\mathbf{I T i p o = p u b l i}$ caciones\&|Funcion=loadContenidoPublicacion\&id=1348

IAvH - Instituto de Investigación de Recursos Biológicos Alexander von Humboldt. 2009. Informe sobre el estado de la biodiversidad en Colombia 2007-2008: piedemonte orinoquense, sabanas y bosques asociados al norte del río Guaviare. Bogotá, Colombia.

Le Treut H, Cubasch S, Ding U, Mauritzen Y, Mokssit C, Peterson A y Prather M. 2007. Historical overview of climate change. In: Solomon S, Qin D, Manning M, Chen Z, Marquis M, Averyt KB, Tignor M y Miller HL (Editores). Climate Change 2007: The Physical Science Basis. Contribution of Working Group I to the Fourth Assessment Report of the Intergovernmental Panel on Climate Change Cambridge University Press, Cambridge, United Kingdom and New York, NY, USA.

Magrin G, Gay García C, Cruz D, et al. 2007 Latin America. En: Parry M, Canziani O, Palutifok J,. van der Linden P y Hanson C. (Editores). Climate Change 2007: Impacts, Adaptation and Vulnerability. Contribution of Working Group II to the Fourth Assessment Report of the Intergovermental Panel on Climate Change. Cambridge, UK: Cambridge University Press. p. 581615.

Maldonado-Ocampo, J, Vari R, Usma J. Checklist of the freshwater fishes from Colombia. Biota Colombiana 2008: 9(2): 143-237

Marín P. 2009. Ficha cambio climático en Colombia. Oficial de Programa de cambio climático y energía. PNUD. p. 2

Martín F. 1989. El carbón vegetal, propiedades y obtención. Agroguías. Editorial Mundiprensa.

MAVDT - Ministerio de Ambiente, Vivienda y Desarrollo Territorial. Relación de emisión de CERs. 2011; [fecha de acceso 0110-2011] URL: http://www.minambiente.gov.co/documentos/
DocumentosAmbiente/cambio_climatico/240211_cers_emitidos.pdf

McNish T. 2007. Las Aves de los Llanos de la Orinoquia. MyB Riqueza Natural.

Meridian Institute. Reducing emissions from deforestation and forest degradation (REDD): An options assessment report. 2009; [fecha de acceso 01-10-2011] URL: http://www.REDD-OAR.org

Metz B, Davidson O, Bosch P, Dave R, Meyer L. (Editores). 2007. Climate Change: Mitigation. Contribution of Working Group III to the Fourth Assessment Report of the Intergovernmental Panel on Climate Change, IPCC, Cambridge University Press, Cambridge, United Kingdom and New York, NY, USA.

Minminas - Ministerio de Minas de Colombia. 2011. Producción anual promedio de crudo en la región de la Orinoquia.

MINAM - Ministerio del Ambiente. 2009. Guía para la elaboración de la Estrategia Regional frente al cambio climático. Ministerio del Ambiente, Perú.

Mittermeir, R, Robles H y Mittermeir C. 1997. Megadiversidad. Los países biológicamente más ricos del mundo. Cemex. México.

MMA. DNP. Ministerio del Medio ambiente. Departamento Nacional de Planeación. 2002. Lineamientos de política de cambio climático. Resumen Ejecutivo.

ONU - Organización da las Naciones Unidas. 2008. Objetivos de desarrollo del Milenio. Informe 2008. Departamento de Asuntos Económicos y Sociales, p. 56.

ONU - Organización da las Naciones Unidas. 2000. Declaración del Milenio. Asamblea General de las Naciones Unidas, p. 10.

ONU - Organización da las Naciones Unidas. 1997. Protocolo de Kyoto de la convención marco de las naciones unidas sobre el cambio climático. Naciones Unidas, p. 25.

Oreskes N. Beyond the Ivory Tower. The scientific consensus on climate change. Science 2004;306(5702):1686

PNUD - Programa de las Naciones Unidas para el Desarrollo. 2009. Cambio Climático. Memorias panel nacional de políticas de pobreza y adaptación al cambio climático en Colombia. Recomendaciones CONPES.

PNUD - Programa de las Naciones Unidas para el Desarrollo. 2008. Programa Conjunto - Fondo para los Objetivos del Desarrollo del Milenio. Perú.

PNUMA - Programa de la Naciones Unidas para el Medio Ambiente. Red de Información en Consumo y Producción Sostenible para América Latina y el Caribe; [fecha de acceso 01-10-2011] URL: http://www.redpycs.net/?modo=biblioteca\&lang=1

PNUMA - Programa de la Naciones Unidas para el Medio Ambiente. 2011. Hacia una economía verde: Guía para el desarrollo sostenible y la erradicación de la pobreza - Síntesis para los encargados de la formulación de políticas.

Parry M, Canziani O, Palutikof J, van der Linden P, Hanson C. (Editores). 2007. Climate Change 2007: impacts, adaptation and vulnerability. contribution of working group ii to the fourth 
assessment report of the intergovernmental panel on climate change. Cambridge University Press, Cambridge, UK.

Postigo, J. 2009. Estrategias de adaptación y gestión frente al cambio climático en tres regiones del sur andino peruano. CEPES.

Paz-Cigarán M, García J. 2006: Cambio climático en el Perú: variable a considerar para el desarrollo sostenible. Tecnología y Sociedad 7, ITDG, Lima, p. 56-68.

Rodríguez-Becerra M y Espinosa G. 2002. Gestión Ambiental en América Latina y el Caribe: evolución, tendencias y principales prácticas. BID.

Rodríguez-Becerra, M. Historia de la política y cooperación ambientales en Colombia y sus consecuencias. Foro Nacional Ambiental y Uniandes. Barranquilla 2005; [Acceso 01-10-11] URL: http://www.uninorte.edu.co/extensiones/IDS/Ponencias/ PONENCIAS\%20AGOSTO\%2024/Historia\%20de\%20la\%20 politica $\% 20 \mathrm{y} \% 20$ cooperacion $\% 20$ ambientales.pdf

Rodríguez-Becerra, M. 2008. La sostenibilidad ambiental. En: León T y Gallini E. (Compiladores). Los biocombustibles en Colombia a debate. Universidad Nacional de Colombia- Colciencias - Cámara de Representantes de la República de Colombia. Bogotá.

Rodríguez-Becerra, M. et al. 2009. La mejor Orinoquía que podemos construir. Elementos para la sostenibilidad ambiental de desarrollo. Corporinoquia. Uniandes. Foro Nacional Ambiental. Friedrich Ebert Stiftung- Colombia.

Romero M, Galindo G, Otero J y Armenteras D. 2004. Ecosistemas de la cuenca del Orinoco colombiano. Instituto de Investigación de Recursos Biológicos Alexander von Humboldt. Bogotá.

Ruiz J. 2010. Cambio climático en temperatura, precipitación y humedad relativa para Colombia usando modelos meteorológicos de alta resolución (Panorama 2011-2100). IDEAM.
SMA - Secretaria do Meio Ambiente do Estado de Sao Paulo. Governo do Estado de Sao Paulo 2012; [fecha de acceso 11-062012] URL: http://www.ambiente.sp.gov.br

Sampaio G, Marengo J, Nobre C. 2008. A atmosfera das mudanças climáticas. En: Buckeridge M. (Editor). Biologia \& Mudanças climáticas no Brasil. Rima Ed. São Paulo, Brasil.

Solomon S, Qin D, Manning M et al (Editores). 2007. Climate Change: The Physical Science Basis. Contribution of Working Group I to the Fourth Assessment Report of the Intergovernmental Panel on Climate Change. Cambridge University Press, Cambridge, United Kingdom and New York, NY, USA.

Stephen P. 2009. A training manual. Introductory course on reducing emissions from Deforestation and Forest Degradation (REDD). The Nature Conservancy, Conservation International, Deutsche Gesellschaft für Technische Zusammenarbeit (GTZ), Rainforest Alliance, and World Wildlife Fund, Inc.

UN - United Nations. 2012. Resilient people, resilient planet: A future worth choosing, Overview. United Nations. SecretaryGeneral's High-Level Panel on Global Sustainability. New York.

UN - United Nations REDD Program. Framework Document 2008; [fecha de acceso 01-10-2011] URL: http://www.un-redd.org/ Publications/tabid/587/Default.aspx

Viloria J. 2009. Geografía económica de la Orinoquía. Documentos de Trabajo de la Economía Regional. Banco de la República. CEER, Cartagena.

Yepes-Mayorga A, Buckeridge M. Respuestas da las plantas ante los factores ambientales del cambio climático global- Revisión. Revista Colombia Forestal, 2011; 14(2): 211-230 